Nervenarzt 2022 · 93:620-623

https://doi.org/10.1007/s00115-021-01205-x

Angenommen: 14. September 2021

Online publiziert: 27. Oktober 2021

(c) Springer Medizin Verlag $\mathrm{GmbH}$, ein Teil von Springer Nature 2021

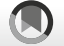

Check for
updates

\section{Seltene Enzephalitis nach Impfung gegen SARS-CoV-2}

Frédéric Zuhorn ${ }^{1}$ Tilmann Graf ${ }^{1}$ - Randolf Klingebiel ${ }^{2}$ · Wolf-Rüdiger Schäbitz ${ }^{1}$. Andreas Rogalewski ${ }^{1}$

${ }^{1}$ Universitätsklinik für Neurologie, Evangelisches Klinikum Bethel, Universitätsklinikum OWL der Universität Bielefeld, Campus Bielefeld-Bethel, Bielefeld, Deutschland

${ }^{2}$ Institut für Diagnostische und Interventionelle Neuroradiologie, Evangelisches Klinikum Bethel, Universitätsklinikum OWL der Universität Bielefeld, Campus Bielefeld-Bethel, Bielefeld, Deutschland

\section{Hintergrund}

Die globale SARS-CoV-2(,severe acute respiratory syndrome coronavirus type $2^{\prime \prime}$ )-Pandemie hat weltweit zu mehr als 222 Mio. bestätigten Infektionen und knapp 4,6 Mio. Todesfällen beigetragen [11]. Es bestehen infolge COVID-19 („,corona virus disease $2019^{\prime \prime}$ ) relevante direkte und indirekte Auswirkungen auf die Behandlung neurologischer Erkrankungen. Eine SARS-CoV-2-Infektion geht mit einer erhöhten Inzidenz neurologischer Manifestationen wie Enzephalopathie und Enzephalomyelitis, ischämischem Schlaganfall und intrazerebralen Blutungen, Anosmie und neuromuskulären Erkrankungen einher [1, 7]. Jede neurologische Manifestation ist mit einem erhöhten Risiko für einen längeren Krankenhausaufenthalt und einer höheren Morbidität und Mortalität im Vergleich zu Patienten ohne neurologische Manifestationen verbunden [7].

Die Schwere der Pandemie hat zu einer beispiellosen Anstrengung geführt, mehrere wirksame Impfstoffe zu entwickeln. Aufgrund hervorragender Sicherheitsund Wirksamkeitsdaten aus klinischen Versuchen wurden mittlerweile mehrere Impfstoffe zugelassen und weltweit knapp 5,6 Mio. Impfdosen appliziert [11]. Neben lokalen und systemischen Nebenwirkungen des ChAdOx1 nCoV-19-Impfstoffs (AZD1222) des Pharmaunternehmens AstraZeneca ohne größere klinische Relevanz erregten in jüngster Zeit immer mehr Berichte über autoimmuninduzierte Thrombozytopenien mit anschließender Thrombose Besorgnis [3, 5, 9]. Kürzlich wurden die ersten drei Fälle postvakzi- naler Enzephalitiden beschrieben [10], die im engen zeitlichen Zusammenhang mit einer ChAdOx1 nCoV-19-Impfung auftraten.

\section{Fallbeschreibung}

Die Patienten (2 Frauen, 1 Mann) im Alter von 21 bis 63 Jahren zeigten das klinische Bild einer Enzephalitis mit Aufmerksamkeits-/Konzentrationsstörungen, Vigilanzstörung, epileptischem Anfall, Aphasie sowie einem Myoklonus-Opsoklonus-Syndrom. Im Liquor zeigte sich eine lymphozytäre Pleozytose und die MRTomographien des Neurokraniums zum Zeitpunkt der Diagnosestellung sowie bei 3-monatiger Verlaufskontrolle ergaben einen Normalbefund. Eine Übersicht der einzelnen Fälle ist in • Tab. 1 dargestellt.

Die Diagnosestellung wurde gestützt durch: 1. den zeitlichen Zusammenhang zwischen Impfung und Symptombeginn, 2. die charakteristischen Symptome, 3. den Ausschluss anderer Ätiologien und 4. das Ansprechen auf eine immunsuppressive Therapie mit Kortikosteroiden.

Nach Ausschluss einer erregerinduzierten Enzephalitis durch umfangreiche Laboruntersuchungen wurden zwei Patienten immunsuppressiv behandelt mit rascher klinischer Besserung. Der dritte Patient lehnte eine immunsuppressive Behandlung $\mathrm{ab}$ und besserte sich spontan. Alle Fälle waren charakterisiert durch insgesamt gutartige Verläufe ohne Folgeerscheinungen. 
Tab. 1 Überblick der berichteten Patienten mit Enzephalitis entsprechend der Publikation nach Zuhorn et al. [10]

\begin{tabular}{|c|c|c|c|}
\hline & Fall 1 & Fall 2 & Fall 3 \\
\hline Alter & 21 Jahre & 63 Jahre & 63 Jahre \\
\hline Geschlecht & Weiblich & Weiblich & Männlich \\
\hline Symptome & $\begin{array}{l}\text { Fieber, Kopfschmerzen, Konzentra- } \\
\text { tions-/Aufmerksamkeitsstörungen, } \\
\text { und epileptischer Anfall }\end{array}$ & $\begin{array}{l}\text { Progrediente Gangunsicherheit, Vigilanz- } \\
\text { störung, Myoklonus-Opsoklonus-Syndrom }\end{array}$ & Fieber und isolierte Aphasie \\
\hline $\begin{array}{l}\text { Symptombeginn (Tage } \\
\text { nach Impfung) }\end{array}$ & 5 & 6 & 8 \\
\hline $\begin{array}{l}\text { MRT Befund bei Aufnah- } \\
\text { me }\end{array}$ & $\begin{array}{l}\text { Keine Enzephalitis, keine Infarkte } \\
\text { und keine Blutung, intrakranielle } \\
\text { Gefäße ohne eine relevante Läsion, } \\
\text { altersentsprechendes cMRT }\end{array}$ & $\begin{array}{l}\text { Kein Hinweis auf eine entzündliche ZNS- } \\
\text { Erkrankung, keine intrakraniellen Metas- } \\
\text { tasen, kein Hinweis auf eine Meningeosis } \\
\text { carcinomatosa, bandförmiges Enhance- } \\
\text { ment im linken Thalamus, korrespondie- } \\
\text { rende Suszeptibilitätsartefakte,z. B. im } \\
\text { Rahmen einer kapillären Teleangiektasie }\end{array}$ & $\begin{array}{l}\text { Altersgerechtes MRT des Neuro- } \\
\text { kraniums ohne sicheres Bildkor- } \\
\text { relat für die aktuelle Klinik, zumal } \\
\text { keine Enzephalitis }\end{array}$ \\
\hline EEG-Befund & $\begin{array}{l}\text { Allgemeinveränderungen mit Theta- } \\
\text { Wellen, keine epilepsietypischen } \\
\text { Potenziale }\end{array}$ & $\begin{array}{l}\text { Allgemeinveränderungen mit Theta- und } \\
\text { Delta-Wellen, keine epilepsietypischen } \\
\text { Potenziale }\end{array}$ & Normales EEG \\
\hline $\begin{array}{l}\text { Liquorbefund } \\
\text { Normalbefund: Leuko- } \\
\text { zyten }<5 / \mu \mathrm{l} \text {, Erythrozy- } \\
\text { ten }<100 / \mu \mathrm{l} \text {, Glukose } \\
\text { im Liquor }<75 \mathrm{mg} / \mathrm{dl} \\
\text { (<4,2 mmol/l), Laktat im } \\
\text { Liquor } 1,70-2,60 \mathrm{mmol} / \mathrm{l} \text {, } \\
\text { Gesamteiweiß im Liquor } \\
180-430 \mathrm{mg} / \mathrm{l} \text {, Albumin im } \\
\text { Liquor }<350 \mathrm{mg} / \mathrm{l}\end{array}$ & $\begin{array}{l}\text { Leukozyten } 46 / \mu l \text { mit lympho- } \\
\text { zytärem Zellbild, Erythrozyten } \\
300 / \mu l, \text { Glukose im Liquor } 50 \mathrm{mg} / \mathrm{dl} \\
\text { (2,8 mmol/l) (64\% L/S-Quotient), } \\
\text { Laktat im Liquor 1,36 mmol/l, Ge- } \\
\text { samteiweiß im Liquor } 233 \mathrm{mg} / \mathrm{l}, \\
\text { Albuminquotient L/S 3,9. Positive } \\
\text { oligoklonale Banden im Serum und } \\
\text { Liquor, systemische Synthese }\end{array}$ & 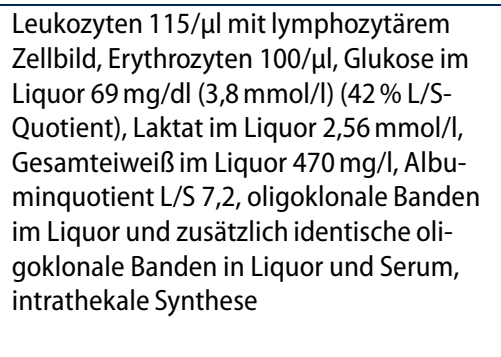 & 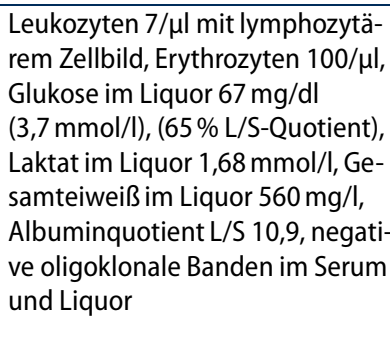 \\
\hline $\begin{array}{l}\text { Standardisierte Blutun- } \\
\text { tersuchungen }\end{array}$ & \multicolumn{3}{|c|}{$\begin{array}{l}\text { Differenzialblutbild, Nieren- und Leberwerte, Schilddrüsenwerte einschl. Schilddrüsenantikörpern, Serumeiweißelektro- } \\
\text { phorese, HIV-Antikörper, Tuberkulose(TB)-QuantiFERON Test, Vaskulitis screen (ANA, Ena-Screen, ANCA-Screen, RF, anti- } \\
\text { ds-DNA, C3c, C4, IgG, IgA, IgM) }\end{array}$} \\
\hline $\begin{array}{l}\text { Standardisierte virolo- } \\
\text { gische Untersuchungen }\end{array}$ & \multicolumn{3}{|c|}{$\begin{array}{l}\text { DNA-PCR für Herpes-Simplex-Virus, Varizella-Zoster-Virus, Zytomegalie- und Epstein-Barr-Virus. } \\
\text { Masern-IgG, Röteln-IgG, Herpes-Simplex-Virus-1/2-IgG, Varizella-Zoster-Virus-IgG }\end{array}$} \\
\hline Antineuronale Antikörper & \multicolumn{3}{|c|}{$\begin{array}{l}\text { GAD65, NMDA- und GABA-B-Rezeptor, IgLON5, AMPA-R Subtyp 2, DPPX, LGI1, CASPR2, Glycinerezeptor, mGluR5, } \\
\text { mGluR1, Amphiphysin, CV2/CRMP5, Ma2/Ta, Hu, Ri, Yo, Zic4, Recoverin, Sox1, Titin, DNER/Tr }\end{array}$} \\
\hline Therapie & Dexamethason & Methylprednisolon & Spontane Remission \\
\hline
\end{tabular}

Tab. 2 Überblick über gemeldete Enzephalitiden als Nebenwirkung nach COVID-19-Impfung aus öffentlich zugänglichen Datenbanken und Schätzung der Häufigkeit

\begin{tabular}{|c|c|c|c|c|}
\hline Institution & Zeitraum & Anzahl an Impfdosen & Berichtete Anzahl an Enzephalitiden & Referenz \\
\hline \multirow[t]{2}{*}{ Gov.uk } & $\begin{array}{l}04.01 .2021 \text { bis } \\
04.08 .2021\end{array}$ & $\begin{array}{l}\text { ChAdOx1 nCoV-19: } \\
\text { 24,8 Mio. 1. Dosis } \\
\text { 23,8 Mio. 2. Dosis }\end{array}$ & $\begin{array}{l}40 \text { Enzephalitiden } \\
1 \text { Bickerstaff-Enzephalitis } \\
2 \text { autoimmune Enzephalitiden } \\
1 \text { limbische Enzephalitis } \\
7 \text { nichtinfektiöse Enzephalitiden }\end{array}$ & \multirow[t]{2}{*}{$\begin{array}{l}\text { https://www.gov. } \\
\text { uk/ }\end{array}$} \\
\hline & $\begin{array}{l}09.12 .2020 \text { bis } \\
04.08 .2021\end{array}$ & $\begin{array}{l}\text { Pfizer-BioNTech mRNA-Vakzin (Comirnaty): } \\
\text { 20,8 Mio. 1. Dosis } \\
\text { 14,7 Mio. 2. Dosis }\end{array}$ & $\begin{array}{l}15 \text { Enzephalitiden } \\
1 \text { autoimmune Enzephalitis } \\
1 \text { limbische Enzephalitis } \\
4 \text { nichtinfektiöse Enzephalitiden }\end{array}$ & \\
\hline $\begin{array}{l}\text { European Medicines } \\
\text { Agency (EMA) }\end{array}$ & $\begin{array}{l}29.01 .2021 \text { bis } \\
10.06 .2021\end{array}$ & $\begin{array}{l}\text { ChAdOx1 nCoV-19: } \\
\text { ca. } 46 \text { Mio. Dosen }\end{array}$ & $\begin{array}{l}33 \text { Enzephalitiden } \\
10 \text { ADEM }\end{array}$ & $\begin{array}{l}\text { https://www.ema. } \\
\text { europa.eu }\end{array}$ \\
\hline \multirow[t]{3}{*}{ Paul-Ehrlich-Institut } & \multirow[t]{3}{*}{$\begin{array}{l}27.12 .2020 \text { bis } \\
30.06 .2021\end{array}$} & $\begin{array}{l}\text { ChAdOx1 nCoV-19: } \\
\text { 11,6 Mio. Dosen }\end{array}$ & 18 Enzephalitiden & \multirow[t]{3}{*}{$\begin{array}{l}\text { https://www.pei. } \\
\text { de }\end{array}$} \\
\hline & & $\begin{array}{l}\text { Pfizer-BioNTech mRNA-Vakzin (Comirnaty): } \\
\text { 54,9 Mio. Dosen }\end{array}$ & 11 Enzephalitiden & \\
\hline & & $\begin{array}{l}\text { COVID-19-Vakzin Moderna (Spikevax): } \\
\text { 6,5 Mio. Dosen }\end{array}$ & Keine Fälle gemeldet & \\
\hline
\end{tabular}




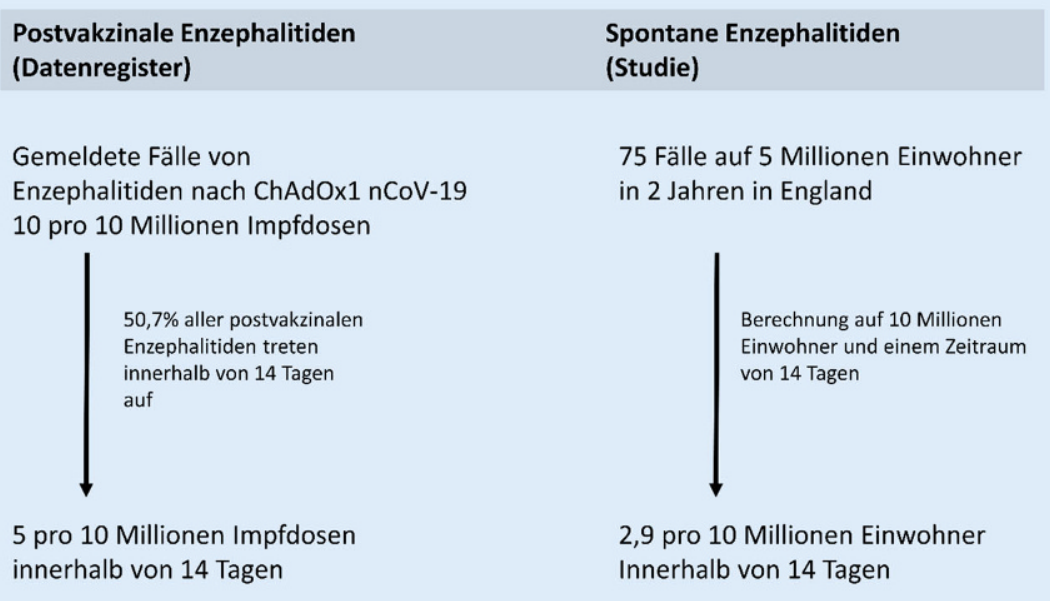

Abb. $1 \Delta$ Gegenüberstellung der Inzidenzberechnung postvakzinaler Enzephalitiden aus den öffentlich zugänglichen Datenregistern (Paul-Ehrlich-Institut, EMA, UK.gov) mit den zu erwartenden spontanen Enzephalitiden ungeklärter Ätiologie in Anlehnung an eine britische Erhebung [4]

\section{Diskussion}

Die Fallserie ist durch das Auftreten von Enzephalitissymptomen innerhalb von 7 bis 11 Tagen nach Impfung gegen SARSCoV-2 mit dem Vakzin ChAdOx1 nCoV-19 gekennzeichnet. Postvakzinale Enzephalitiden stellen eine bekannte Entität dar. In einem Erhebungszeitraum von 20 Jahren wurden in den Vereinigten Staaten 1396 Fälle postvakzinaler Enzephalitis nach Impfungen gegen Hepatitis B (354 Fälle), Influenza (208 Fälle), Masern, Mumps und Röteln (208 Fälle) und Haemophilus influenzae Typ B (120 Fälle) berichtet [8]. Bei 708 Patienten (50,7\%) trat die Enzephalitis innerhalb von 14 Tagen nach der Impfung auf. Als Ursache der postvakzinalen Neuroinflammation wird die starke Expression proinflammatorischer Zytokine sowie eine T-Zell-Reaktion vermutet. Dies wurde auch für den Impfstoff ChAdOx1 nCoV19 nachgewiesen [2]. Nach der Impfung werden Antigene als potenzielle Krankheitserreger erkannt. Induktion und Transkription zahlreicher Zielgene führt zur Synthese und Freisetzung pyrogener Zytokine in den Blutkreislauf. Diese imitieren die Reaktion einer natürlichen Infektion. Nach Stimulation setzt das Immunsystem eine komplexe Kaskade des genuinen Immunsystems in Gang. Entzündungsmediatoren und -metabolite im Blutkreislauf können andere Körpersysteme beeinträchtigen und systemische Nebenwirkungen hervorrufen. Schließlich wird durch Aktivierung der Mikroglia eine Neuroinflammation verursacht [6].

Über das Auftreten von Enzephalitiden nach einer Impfung mit ChAdOx1 nCoV19 wird in mehreren öffentlich zugänglichen Datenregistern berichtet (• Tab. 2). Insgesamt wurden bisher 102 Enzephalitisfälle bei 106,2 Mio. Impfdosen ChAdOx1 nCoV-19 gemeldet. Anhand der gemeldeten Ereignisse und der in diesem Zeitraum verabreichten Impfungen kann die Inzidenz auf etwa 10 pro 10 Mio. Impfdosen geschätzt werden.

Eine vergleichbare Analyse des mRNAImpfstoffs des Pharmaunternehmens Pfizer-BioNTech (Comirnaty) ergab nur 32 gemeldete Enzephalitisfälle bei 90,4 Mio. verabreichten Impfdosen. Die geschätzte Inzidenz liegt damit bei etwa 4 von 10Mio. Impfdosen, signifikant niedriger im Vergleich zu ChAdOx1 nCoV-19 (ChAdOx1 nCoV-19 vs. Comirnaty; Pearson's $X^{2}=26,4$; $p<0,001)$.

Es erfolgte eine Gegenüberstellung der Inzidenzen der möglichen postvakzinalen Enzephalitiden mit spontan auftretenden Enzephalitiden ungeklärter Ätiologie. Dafür war die Definition einer vergleichbaren Zeitspanne erforderlich. In den gemeldeten Impfregisterdaten wurde durch das Robert-Koch-Institut ein Auftreten innerhalb von maximal 91 Tagen nach Impfung berichtet, während die EMA und die englischen Registerdaten keine Zeitspan- ne benannten. Entsprechend der US-Erhebung von 1396 Enzephalitisfällen mit $50,7 \%$ aller aufgetretenen Fälle innerhalb von 14 Tagen [8] definierten wir dieses Intervall als relevanten Zeitraum nach erfolgter Impfung. Dies entsprach für die in den öffentlich zugänglichen Datenregistern gemeldeten Fälle nach Impfung mit ChAdOx1 nCoV-19 einer Inzidenz der möglichen postvakzinalen Enzephalitis von 5 pro 10Mio. Impfdosen.

Dieser Anzahl wurde die zu erwartende Anzahl spontan auftretender Enzephalitiden ungeklärter Ätiologie entsprechend der britischen Studie mit 75 Fällen pro 5 Mio. Einwohner in einem Zeitraum von 2 Jahren gegenübergestellt [4]. Berechnet auf ein Zeitintervall von 14 Tagen und 10 Mio. Personen wäre somit eine Rate spontan auftretender ungeklärter Enzephalitisfälle von 2,9 pro 10 Mio. Personen zu erwarten.

Ein Vergleich zwischen erhobenen Fällen öffentlich zugänglicher Impfregisterdaten mit den Daten einer Studie zu spontan auftretenden Fällen einer Enzephalitis ungeklärter Ätiologie kann als Schätzung der Fälle angesehen werden (• Abb. 1). Diese stellt aufgrund der Methodik allerdings keine genaue Analyse dar, was als Limitation dieser Analyse anzusehen ist.

Anzunehmen ist aber, dass nicht alle Fälle gemeldet wurden und daher die tatsächliche Inzidenz der postvakzinalen Enzephalitis höher ist. Eine systematische Erhebung und Meldung mutmaßlicher postvakzinaler Enzephalitiden wäre für eine belastbarere Datenanalyse wünschenswert.

\section{Fazit}

Die Komplikation einer Enzephalitis nach einer ChAdOx1 nCoV-19-Impfung ist auf der Basis vorliegender Daten sehr selten. Es finden sich Hinweise, dass das Risiko, eine postvakzinale Enzephalitis zu entwickeln, nach einer Impfung mit ChAdOx 1 nCoV-19 leicht erhöht ist. Auch wenn bisher nur der zeitliche Zusammenhang zwischen Impfung und Enzephalitis dargelegt werden kann, sind das gehäufte Auftreten einer Enzephalitis sowie eine unterschiedliche Inzidenz in Abhängigkeit des verwendeten Impfstoffs erkennbar. Eine systematische Erhebung und Meldung auftretender Fälle einer mutmaßlich postvakzinalen Enze- 
phalitis wäre für eine belastbarere Datenanalyse wünschenswert. Der Nutzen der Impfung überwiegt eindeutig die Risiken.

\section{Korrespondenzadresse}

\section{Dr. med. Frédéric Zuhorn}

Universitätsklinik für Neurologie, Evangelisches Klinikum Bethel, Universitätsklinikum OWL der Universität Bielefeld, Campus Bielefeld-Bethel Burgsteig 13, 33617 Bielefeld, Deutschland frederic.zuhorn@evkb.de

\section{Einhaltung ethischer Richtlinien}

Interessenkonflikt. F. Zuhorn, T. Graf, R. Klingebiel, W.-R. Schäbitz und A. Rogalewski geben an, dass kein Interessenkonflikt besteht.

Von allen drei Patienten erhielten wir die schriftliche Einverständniserklärung, dass die Beschreibungen der Erkrankung und die Abbildungen anonym veröffentlicht werden dürfen.

\section{Literatur}

1. Berlit P, Bösel J, Gahn G et al (2020) "Neurological manifestations of COVID-19" - guideline of the German society of neurology. Neurol Res Pract
21(2):1-14. https://doi.org/10.1186/\$42466-02000097-7

2. Ewer KJ, Barrett JR, Belij-Rammerstorfer $S$ et al (2021) T cell and antibody responses induced by a single dose of ChAdOx1 nCoV-19 (AZD1222) vaccine in a phase $1 / 2$ clinical trial. Nat Med 27:270-278. https://doi.org/10.1038/s41591020-01194-5

3. Graf T, Thiele T, Klingebiel R et al (2021) Immediate high-doseintravenousimmunoglobulinsfollowed by direct thrombin-inhibitor treatment is crucial for survival in Sars-Covid-19-adenoviral vector vaccine-induced immune thrombotic thrombocytopenia VITT with cerebral sinus venous and portal vein thrombosis. J Neurol. https://doi.org/ 10.1007/s00415-021-10599-2

4. Granerod J, Ambrose H, Davies N et al (2010) Causes of encephalitis and differences in their clinical presentations in England: a multicentre, population-based prospective study. Lancet Infect Dis 10:835-844. https://doi.org/10.1016/S14733099(10)70222-X

5. Greinacher A, Thiele T, Warkentin TE et al (2021) Thrombotic thrombocytopenia after ChAdOx1 nCov-19vaccination. NEngl J Med. https://doi.org/ 10.1056/nejmoa2104840

6. Hervé C, Laupèze B, Del Giudice G et al (2019) The how's and what's of vaccine reactogenicity. NPJ Vaccines 4:39. https://doi.org/10.1038/s41541019-0132-6

7. Leven Y, Bösel J (2021) Neurological manifestations of COVID-19-an approach to categories of pathology. Neurol Res Pract 3(1):39. https://doi. org/10.1186/s42466-021-00138-9

8. Al Qudah Z, Abukwaik W, Patel H, Souayah N (2012) Encephalitis after vaccination in United
States. A report from the CDC/FDA vaccine adverse event reporting system. [1990-2010]. Neurology 78:P03.151

9. Schultz NH, Sørvoll IH, Michelsen AE et al (2021) Thrombosis and thrombocytopenia after ChAdOx 1 nCoV-19 vaccination. N Engl J Med. https://doi. org/10.1056/nejmoa2104882

10. Zuhorn F, Graf T, Klingebiel R et al (2021) Postvaccinal encephalitis after ChAdOx1 nCov-19. Ann Neurol. https://doi.org/10.1002/ANA.26182

11. Johns Hopkins Coronavirus Resource Center (2021) COVID-19 map. https://coronavirus.jhu.edu/map. html.Zugegriffen: 9. Sept. 2021

\section{Hier steht eine Anzeige.}

\section{Springer}

